\title{
On the origin of correlated X-ray/VHE emission from LS I +61303
}

\author{
V. Zabalza ${ }^{1}$, J. M. Paredes ${ }^{1}$, and V. Bosch-Ramon ${ }^{2}$
}

\author{
1 Dept. d'Astronomia i Meteorologia, Institut de Ciències del Cosmos (ICC), Universitat de Barcelona (IEEC-UB), \\ Martí i Franquès, 1, 08028, Barcelona, Spain \\ e-mail: vzabalza@am.ub.es \\ 2 Dublin Institute for Advanced Studies, 31 Fitzwilliam Place, Dublin 2, Ireland
}

Received 10 July 2010 / Accepted 16 November 2010

\section{ABSTRACT}

\begin{abstract}
Context. The MAGIC collaboration recently reported correlated X-ray and very high-energy (VHE) gamma-ray emission from the gamma-ray binary LS I +61 303 during $\sim 60 \%$ of one orbit. These observations suggest that the emission in these two bands has its origin in a single particle population.

Aims. We aim at improving our understanding of the source behaviour by explaining the simultaneous X-ray and VHE data through a radiation model.

Methods. We use a model based on a one zone population of relativistic leptonic particles at the position of the compact object and assume dominant adiabatic losses. The adiabatic cooling time scale is inferred from the X-ray fluxes.

Results. The model can reproduce the spectra and light curves in the X-ray and VHE bands. Adiabatic losses could be the key ingredient to explain the X-ray and partially the VHE light curves. From the best-fit result we obtain a magnetic field of $B \simeq 0.2 \mathrm{G}$, a minimum luminosity budget of $\sim 2 \times 10^{35} \mathrm{erg} \mathrm{s}^{-1}$ and a relatively high acceleration efficiency. In addition, our results seem to confirm that the $\mathrm{GeV}$ emission detected by Fermi does not come from the same parent particle population as the X-ray and VHE emission. Moreover, the Fermi spectrum poses a constraint on the hardness of the particle spectrum at lower energies. In the context of our scenario, more sensitive observations would allow us to constrain the inclination angle, which could determine the nature of the compact object.
\end{abstract}

Key words. stars: individual: LS I +61 303 - stars: emission line, Be - X-rays: binaries - radiation mechanisms: non-thermal

\section{Introduction}

LS I +61303 is one of the few X-ray binaries (along with PSR B1259-63, LS 5039 and probably Cygnus X-1) that have been detected in very high-energy (VHE) gamma rays ${ }^{1}$. This source is a high-mass X-ray binary located at a distance of $2.0 \pm 0.2 \mathrm{kpc}$ (Frail \& Hjellming 1991) and contains a compact object with a mass between 1 and $4 M_{\odot}$ orbiting the main Be star every $26.4960 \pm 0.0028 \mathrm{~d}$ (Gregory 2002) in an eccentric orbit (see Aragona et al. 2009, and Fig. 1). The main B0 Ve star has a mass of $M_{\star}=(12.5 \pm 2.5) M_{\odot}$, a radius of $R_{\star} \approx 10 R_{\odot}$ and a bolometric luminosity of $10^{38} \mathrm{erg} / \mathrm{s}$ (Casares et al. 2005). Observations of persistent jet-like features in the radio domain at $\sim 100$ milliarcsecond (mas) scales prompted a classification of the source as a microquasar (Massi et al. 2004; see also Massi \& Kaufman Bernadó 2009, for a radio spectral discussion), but later observations at $\sim 1-10$ mas scales, covering a whole orbital period, revealed a rotating elongated feature that was interpreted as the interaction between a pulsar wind and the stellar wind (Dhawan et al. 2006). In the X-ray domain, LS I +61303 shows an orbital periodicity (Paredes et al. 1997) with quasi-periodic outbursts in the phase range 0.4-0.8. The source also shows short-term flux and spectral variability on time scales of kiloseconds (Sidoli et al. 2006; Rea et al. 2010). LS I +61303 has been detected in the VHE domain by MAGIC (Albert et al. 2006) and VERITAS (Acciari et al. 2008). It shows a periodic behaviour (Albert et al. 2009) with maxima occurring around phase 0.6-0.7 and non-detectable flux around

${ }^{1}$ HESS J0632+057 is a variable galactic VHE source thought to be a binary, but no orbital period has been found yet (Hinton et al. 2009; Falcone et al. 2010). periastron $\left(\phi_{\text {per }}=0.275\right)$. However, it must be noted that since the beginning of 2008 there have been no reports of VHE detection around apastron even though VERITAS performed several observation campaigns (Holder 2010). A recent detection of the source around periastron (Ong 2010) indicates that its VHE orbital light curve might not be stable over long periods. Recently LS I +61303 was detected by the high-energy (HE) gamma-ray instrument Fermi/LAT (Abdo et al. 2009). Its emission at GeV energies was found to be anti-correlated in phase with the X-ray and VHE emission and presented a spectrum compatible with a power law and an exponential cutoff at $\sim 6 \mathrm{GeV}$. This spectrum is reminiscent of pulsar magnetospheric emission, but no pulsations were found and no orbital variability would in principle be expected from this kind of emission. Since March 2009 the source shows a higher phase-averaged flux than before and the orbital modulation has significantly diminished (Dubois 2010). This change in GeV behaviour, as well as the change in the VHE light curve, indicates that a long term variability effect may be at play. It is not yet clear, however, whether there is any relation to the $\sim 4.6$ yr superorbital modulation of the radio peak (Paredes 1987; Gregory 1999).

Two scenarios have been proposed to account for the observational features of LS I +61303 . The microquasar scenario requires accretion onto the compact object and the formation of a jet in which particles are accelerated and emit up to VHE gamma-rays through upscattering of stellar photons (e.g. Bosch-Ramon \& Paredes 2004; Bosch-Ramon et al. 2006b). In the pulsar wind zone scenario, the compact object is a young pulsar and particles are accelerated at the interaction zone between the pulsar wind and the stellar wind (Maraschi \& Treves 1981; Dubus 2006). The stellar wind is thought to be formed by 


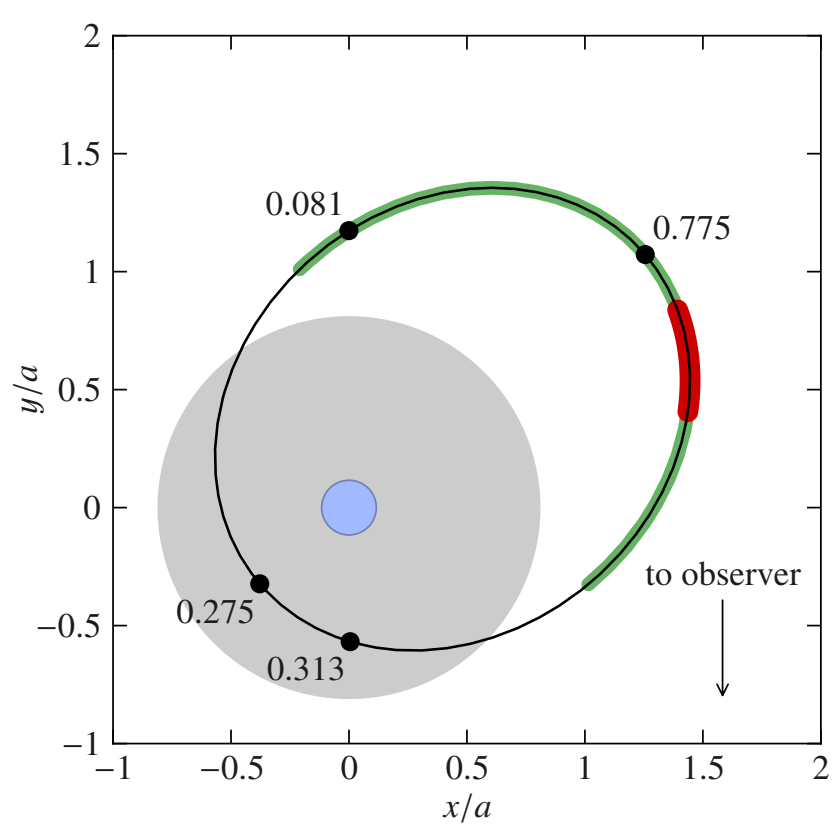

Fig. 1. Orbital geometry of LS I +61 303 looking down on the orbital plane showing the orbit of the compact object around the Be star in units of the orbital semi-major axis. The positions labeled with the orbital phase correspond to periastron, apastron, and inferior and superior conjunctions. The X-ray/VHE multiwavelength campaign covered the phase range shown in green $(0.43<\phi<1.13)$, while the phase range of the VHE emission outburst is shown in red $(0.6<\phi<0.7)$. The Be star (blue circle) and the equatorial wind (grey circle) are shown assuming an equatorial disk radius of $r_{\mathrm{d}}=7 R_{\star}$.

a fast, low-density polar wind and a slow, high-density equatorial decretion disc, which may be strongly truncated and have its properties disturbed by the compact object (Romero et al. 2007). On the other hand, the characteristics of the interaction of pulsar winds or microquasar jets with a massive star wind are not well constrained (but see the studies by, e.g., Bogovalov et al. 2008; Perucho \& Bosch-Ramon 2008). Doppler boosting effects could be present both for a microquasar jet and a shocked pulsar wind outflow (e.g. Khangulyan et al. 2008a; Dubus et al. 2010), but its effects in both scenarios are difficult to assess because of our lack of knowledge on the geometry and orientation of the emitter. Finally, the uncertainty in the orbital geometry, in particular the inclination, also affects the ability of models to recover the physical parameters of the emitter (SierpowskaBartosik \& Torres 2009). All this shows the difficulties of the study of gamma-ray binaries.

Because of the many factors involved, simultaneous Xray/VHE observations are a useful mean to start to disentangle the physics of the high-energy emission in gamma-ray binaries. However, the combined effect of short-term variability in the X-ray domain and night-to-night variability in the VHE domain has precluded a clear detection of X-ray/VHE emission correlation from archival observations. In 2007, a campaign of simultaneous observations with the MAGIC Cherenkov telescope and the XMM-Newton and Swift X-ray satellites revealed a correlation between the X-ray and VHE bands (Anderhub et al. 2009). The suggestion that the emission in both energy bands comes from the same population of accelerated particles (Anderhub et al. 2009, see discussion) makes these observations an ideal data set to test the properties of the X-ray/VHE emitter in LS I +61303 . It is worth noting that the need of only one leptonic population to explain the X-ray and the VHE emission would be against lepto-hadronic models (e.g. Chernyakova et al. 2006).

In this work, we present the results of the application of a leptonic one zone model to explain the measured light curves and spectra from these observations. Our goal is to obtain the physical parameters of the emitter robustly by making as few assumptions as possible.

\section{Simultaneous X-ray/VHE observations}

LS I +61303 was observed during $\sim 60 \%$ of an orbital period simultaneously in the VHE and X-ray bands in September 2007 (Anderhub et al. 2009). During a first part of the campaign $(0.43<\phi<0.7)$, the MAGIC Cherenkov telescope observed the source for three hours every night and simultaneous XMM-Newton observations were performed. During a second part $(0.71<\phi<1.13)$ the X-ray observations were performed with Swift/XRT. The observation times were of about $15 \mathrm{ks}$ for the XMM-Newton observations and of about $3 \mathrm{ks}$ for the Swift/XRT observations. The longer observations and higher effective area of XMM-Newton resulted in much higher statistics than the measurements from Swift/XRT. To take into account the variability that the source shows on short time scales in the X-ray band when comparing these fluxes to the VHE measurements, the rms count rate variability of the X-ray light curve was considered in addition to the statistical uncertainty in the flux measurement to obtain realistic flux uncertainties (see Anderhub et al. 2009, for details). Using this method, the X-ray flux uncertainties take values between $6 \%$ and $25 \%$ of the flux. The simultaneous campaign revealed very similar light curves in the X-ray and VHE bands: a first outburst at $\phi=0.62$ with a rise time below $20 \mathrm{~h}$ and a decay of about two days; and a second broader outburst spanning a few days between phases 0.8 and 1.1 with lower peak flux than the first. The correlation coefficient between the two bands for the first outburst was found to be $r=0.97$, whereas taking all simultaneous observations lowered the value to $r=0.81$.

Although all X-ray observations resulted in a clear detection, some of the VHE flux measurements have a significance below $2 \sigma$. For these cases we will consider the 95\% CL (confidence level) upper limits calculated by Jogler (2009). The detailed fluxes and uncertainties of the X-ray and VHE light curves may be found in Tables 1 and 2 of Anderhub et al. (2009) and the VHE upper limits in Table A.1 of Jogler (2009).

The sensitivity of VHE observations precludes obtaining nightly spectra, and only a spectrum combining the observations with phases between 0.6 and 0.7 was published by Anderhub et al. (2009). In order to obtain a simultaneous SED, we extracted an XMM-Newton spectrum of the source for the three X-ray observations that correspond to the MAGIC spectrum. We filtered the data using SAS v10.0 and extracted three individual spectra from the pn instrument. We used the SAS tool efluxer to convert the spectra from counts vs. channel into physical units (flux density vs. energy), averaged the three unbinned spectra and then grouped the bins to a signal-to-noise ratio of 20 . In order to compare the measured spectrum to the computed intrinsic spectrum, we deabsorbed the former using the wabs FORTRAN subroutine provided with Xspec v12.0 (Arnaud 1996). We used a column density of $N_{\mathrm{H}}=5 \times 10^{21} \mathrm{~cm}^{-2}$ obtained from the average of the individual fits of an absorbed power law to the three observations. This column density is consistent with that of the ISM alone (Paredes et al. 2007). 


\section{Model description}

The discovery of a correlation between the X-ray and VHE bands points towards a common mechanism of emission modulation at both bands. In a leptonic scenario, the fast and simultaneous changes in flux in both bands indicate that the modulation mechanism has to directly affect the emission level of the Inverse Compton (IC) and synchrotron processes. Assuming constant injection, a way to obtain correlated X-ray and VHE emission is through a modulation of the number of emitting particles by dominant adiabatic losses, which would be ultimately related to the (magneto)hydrodynamical processes in the accelerator and emitter regions. These processes may be related, for instance, to the interaction of the pulsar wind or the black hole jet with the stellar wind of the massive companion. The hard X-ray spectrum with photon index $\Gamma \approx 1.5$ also points towards dominant adiabatic losses, which imply an injection electron index of $\alpha_{\mathrm{e}} \approx 2$. In the regime of dominant adiabatic losses the emitted X-ray flux is proportional to the number of emitting particles, so the orbital dependency of adiabatic losses can be inferred from the X-ray light curve. Dominant adiabatic losses are not uncommon when modelling gamma-ray binaries. They have also been invoked to explain the variations of the Xray and VHE fluxes in the gamma-ray binaries PSR B1259-63 and LS 5039 by Khangulyan et al. (2007) (see also Kerschhaggl 2011) and Takahashi et al. (2009), respectively. We note that, as explained in Sect. 3.4, the IC emission will be additionally modulated by the changes in seed photon density along the orbit as well as the geometrical effects of the location of the emitter along the orbit.

For the sake of simplicity, and given how little we know about the exact properties of the particle accelerator and the nonthermal emitter in LS I +61 303, we will here assume a constant particle injection spectrum and emitter magnetic field along the orbit. We will also adopt one leptonic population cooling down under variable adiabatic losses at the location of the emitter, which will be approximated as homogeneous and point-like.

The orbital parameters were adopted from Aragona et al. (2009) with an inclination angle of $i=45^{\circ}$. We will also discuss other possibilities regarding the inclination angle, in particular the extremes of the allowed range $15^{\circ} \lesssim i \lesssim 60^{\circ}$ considered by Casares et al. (2005). A sketch of the orbital configuration of LS I +61303 can be seen in Fig. 1 with the phase ranges of the simultaneous X-ray/VHE campaign indicated. The equatorial wind disk in binaries of the characteristics of LS I +61 303 is expected to be truncated at a radius of $r_{\mathrm{d}}=5 R_{\star}$ (Grundstrom et al. 2007). However, the variability in the equivalent width of the $\mathrm{H} \alpha$ line indicates that its radius can vary between $4.5 R_{\star}$ and $7 R_{\star}$ (Zdziarski et al. 2010; Sierpowska-Bartosik \& Torres 2009). A variable red shoulder in the $\mathrm{H} \alpha$ line (McSwain et al. 2010) could point towards the creation of a tidal stream in the equatorial disk because of the proximity of the compact at periastron that eventually falls back onto the disk at later phases. Even at its largest possible radius of $7 R_{\star}$, as assumed in Fig. 1, the equatorial disk does not affect the phase ranges of the observations we are considering, and thus we will not include it in our model.

\subsection{Adiabatic cooling}

One of the remarkable features of the VHE light curve of LS I +61303 is the lack of detectable emission during periastron (Albert et al. 2009). IC emission is very effective at this phase owing to the high stellar photon density, so the lack of emission means that either it is strongly absorbed or that the number of accelerated electrons falls drastically. However, the angular dependency of the pair production process, $\propto(1-\cos \psi)$ for the interaction rate and $\propto(1-\cos \psi)^{-1}$ for the gamma-ray energy threshold (where $\psi$ is the interaction angle of the incoming photons), implies that absorption is very low for phases immediately after periastron even under the dense seed photon field of the star (see Sect. 3.5 for details on the calculation of pair production absorption). Inverse Compton emission also has a dependency on the angle between the seed photon and the accelerated electron and would be lower during these phases, but this effect is diminished at higher energies when scattering takes place in the deep Klein-Nishina regime (see Fig. 5 in Khangulyan et al. 2008b). A reduction in the number of accelerated particles is required to explain the lack of VHE emission around periastron. Although the X-ray flux during periastron is around half of the peak flux during the periodic outbursts, the non-detection of VHE emission during periastron places an upper limit at only a $10 \%$ of the outburst peak VHE flux. Taking all this into account, we will assume that in the context of one electron population the X-ray/VHE correlation is only valid for the $\mathrm{X}$-ray excess flux above a certain quiescent or pedestal emission. Therefore, the number of emitting particles responsible for this excess X-ray flux is lower and their VHE emission remains at non-detectable levels around periastron. This reasoning is supported by the X-ray/VHE correlation found by Anderhub et al. (2009), in which the constant term of the linear correlation is $\left(12.2_{-1.0}^{+0.9}\right) \times 10^{-12} \mathrm{erg} \mathrm{cm}^{-2} \mathrm{~s}^{-1}$. Considering that some of the $\mathrm{X}$-ray observations are below this constant term, we took the pedestal flux value to be $F_{\text {ped }}=11.5 \times 10^{-12} \mathrm{erg} \mathrm{cm}^{-2} \mathrm{~s}^{-1}$.

As mentioned above, a scenario with dominant adiabatic losses is a very good candidate mechanism to explain the correlation characteristics of the source. When considering a system with constant magnetic field and constant injected electron spectrum, the only modulation in synchrotron radiation is due to the modulation of the dominant cooling process that results in a modulation of the (evolved) stationary electron distribution. For dominant adiabatic losses and a constant injection rate, the X-ray flux will be proportional to the adiabatic cooling time $t_{\mathrm{ad}}$. A consistent calculation of $t_{\mathrm{ad}}$ would require knowledge of the exact nature of the source and the (magneto)hydrodynamical processes that take place there. One can however infer $t_{\text {ad }}$ along the orbit by taking it proportional to the excess flux above the pedestal $F_{\mathrm{X}}-F_{\text {ped }}$, which is independent of the electron energy. For the phases covered by the X-ray observations we derived a smooth curve following the behaviour of the X-ray fluxes. For the phases outside the campaign coverage we assumed a flat light curve consistent with the first four observations at phases 0.43 to 0.59 and the last observation at phase 0.13 . Choosing the ratio $t_{\text {ad }} /\left(F_{X}-F_{\text {ped }}\right)$ as small as possible while keeping the adiabatic losses dominant allows us to put a lower limit on the injected luminosity budget. This choice of the ratio $t_{\mathrm{ad}} /\left(F_{\mathrm{X}}-F_{\text {ped }}\right)$ yields adiabatic loss time scales of between 35 and 500 s. Figure 2 shows the orbital evolution of the cooling time scales inferred for adiabatic and calculated for IC and synchrotron losses. The adiabatic cooling time is independent of the electron energy, but for synchrotron and IC the cooling time scales are given at $E_{\mathrm{e}}=10^{12} \mathrm{eV}$. This energy corresponds to electrons that emit in the X-ray band through synchrotron and in the VHE band through IC.

\subsection{Particle energy distribution}

The radiation emitted by a population of non-thermal particles arises from the contribution of electrons of different ages. 


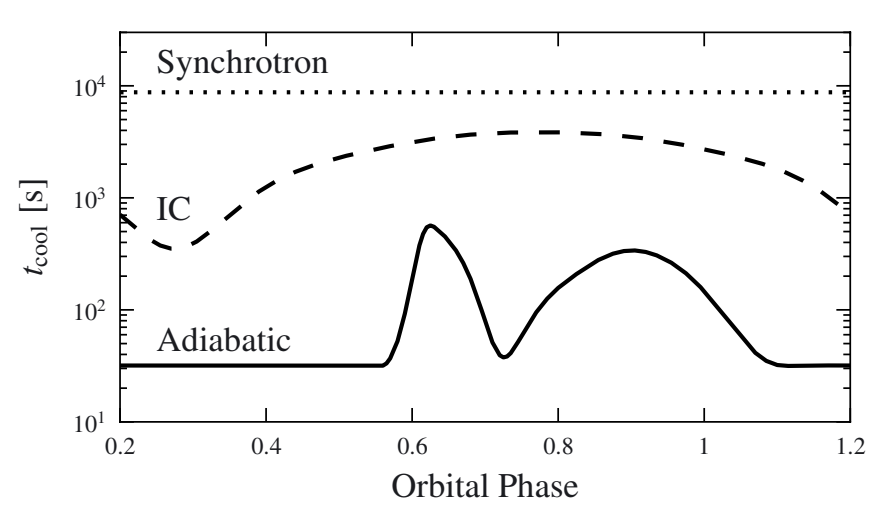

Fig. 2. Cooling times along the orbit for adiabatic (solid), synchrotron (dotted) and IC (dashed). The synchrotron and IC cooling times are shown at $E_{\mathrm{e}}=10^{12} \mathrm{eV}$. This energy corresponds to electrons that emit in the X-ray band through synchrotron and in the VHE band through IC.

As electrons loose energy through radiative or adiabatic cooling processes, an evolved particle energy distribution, different from the injected particle spectrum $(Q(\gamma)$, taken here constant in time), arises. The evolved particle spectrum $n\left(\gamma_{\mathrm{e}}, t\right)$ can be obtained from $Q(\gamma)$ through the following equation (Ginzburg \& Syrovatskii 1964):

$\frac{\partial n\left(\gamma_{\mathrm{e}}, t\right)}{\partial t}+\frac{\partial \dot{\gamma} n\left(\gamma_{\mathrm{e}}, t\right)}{\partial \gamma_{\mathrm{e}}}=Q\left(\gamma_{\mathrm{e}}\right)$

where $\dot{\gamma}$ is the sum of the energy loss rates for IC, synchrotron and adiabatic processes and $Q\left(\gamma_{\mathrm{e}}\right)$ is the acceleration rate. Since little is known about the acceleration process responsible for particle acceleration in LSI +61303 , we assume a constant particle injection spectrum along the orbit. We adopt a phenomenological electron injection spectrum $Q\left(\gamma_{\mathrm{e}}\right)$ consisting of a power law function with an exponential high energy cutoff: $Q\left(\gamma_{\mathrm{e}}\right)=Q_{0} \gamma_{\mathrm{e}}^{-\alpha_{\mathrm{e}}} \exp \left(-\gamma_{\mathrm{e}} m c^{2} / E_{\mathrm{e}, \max }\right)$. The maximum electron energy $E_{\mathrm{e}, \max }$ is obtained from the balance of acceleration and energy loss rates, thus establishing a cutoff in the accelerated particle energy spectrum (see Sect. 3.3); $\alpha_{\mathrm{e}}$ is typically $\sim 2$ in the context of non-relativistic first order Fermi acceleration (see, e.g., Drury 1983); and $Q_{0}$ is the normalization of the function, which we keep constant along the orbit.

For LS I +61 303 the adiabatic and radiative cooling time scales of high-energy electrons are much shorter than the orbital period, and thus we can calculate the steady-state electron energy distribution along the orbit omitting the first term in Eq. (1) and solving for $n\left(\gamma_{\mathrm{e}}\right)$ :

$n\left(\gamma_{\mathrm{e}}, \phi\right)=\frac{1}{|\dot{\gamma}(\phi)|} \int_{\gamma_{\mathrm{e}}}^{\gamma_{\mathrm{e}}^{\max }} Q\left(\gamma^{\prime}\right) \mathrm{d} \gamma^{\prime}$,

The resulting $n\left(\gamma_{\mathrm{e}}, \phi\right)$ is the evolved particle spectrum for which the emitted radiation properties are calculated along the orbit.

\subsection{Maximum electron energy}

The VHE gamma-ray energy spectrum of LS I +61 303 extends up to $E_{\gamma} \sim 10 \mathrm{TeV}$ (see Acciari et al. 2009) without a break in the spectrum, indicating that electrons must be accelerated up to energies beyond $10 \mathrm{TeV}$. The acceleration of electrons has to be faster than the cooling times of the different cooling mechanisms so that the electrons can reach these very high energies.

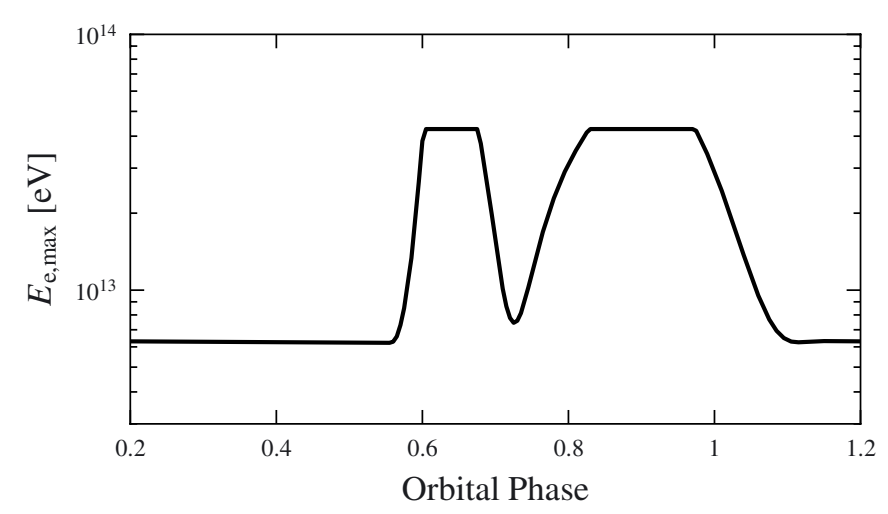

Fig. 3. Maximum electron energy $E_{\mathrm{e}, \max }$ along the orbit taking $\eta=10$ (see text for details).

The maximum electron energy will be that for which $t_{\mathrm{acc}}=t_{\mathrm{cool}}$. The acceleration time of electrons can be characterized as

$t_{\mathrm{acc}}=\eta r_{\mathrm{L}} / c \approx 0.11 \eta E_{\mathrm{TeV}} B_{\mathrm{G}}^{-1} \mathrm{~s}$,

where $r_{\mathrm{L}}=E / e B$ is the Larmor radius, $B_{\mathrm{G}}$ is the magnetic field in Gauss and $E_{\mathrm{TeV}}$ is the electron energy in TeV. The dimensionless constant $\eta \geq 1$ indicates the efficiency of the acceleration process, where $\eta \rightarrow 1$ corresponds to the maximum possible acceleration rate allowed by classical electrodynamics and $\eta=2 \pi\left(v_{\mathrm{s}} / c\right)^{-2}$ for parallel non-relativistic diffusive shocks in the Bohm regime (Drury 1983).

If electron energy losses are dominated by adiabatic cooling, the maximum energy that electrons will reach is given by $t_{\mathrm{acc}}=$ $t_{\mathrm{ad}}$, which results in

$E_{\mathrm{e}, \max } \approx 9 B_{\mathrm{G}} t_{\mathrm{ad}} \eta^{-1} \mathrm{TeV}$.

However, at some points along the orbit in which adiabatic losses are smaller, synchrotron losses may dominate at high energies, since $t_{\mathrm{syn}} \propto E_{\mathrm{e}}^{-1}$ whereas the adiabatic cooling time is constant with electron energy. In these cases, with a synchrotron cooling time described by

$t_{\mathrm{syn}} \approx 400 E_{\mathrm{TeV}}^{-1} B_{\mathrm{G}}^{-2} \mathrm{~s}$,

the balance $t_{\mathrm{acc}}=t_{\mathrm{syn}}$ results in a maximum energy of

$E_{\mathrm{e}, \max } \approx 60 B_{\mathrm{G}}^{-1 / 2} \eta^{-1 / 2} \mathrm{TeV}$.

Inverse Compton energy losses at the relevant energies are smaller than to the synchrotron and adiabatic losses. At each point along the orbit, all relevant time scales are evaluated and the corresponding $E_{\mathrm{e}, \max }$ is obtained, which in this work characterizes the position of the cutoff in the injected particle spectrum through an exponential cutoff. The adiabatic cooling times inferred from the X-ray light curve range from a few tens to a few hundred seconds. When considering the requirement that acceleration takes place up to electron energies of $10 \mathrm{TeV}$, we can see that the accelerator required must be quite efficient with $\eta \approx 7-130$ depending on the orbital phase. Since we are assuming that the injection is constant, we also expect the acceleration rate to be constant and have chosen a value of $\eta=10$ to perform the energy cutoff calculations along the orbit, which would correspond to acceleration in a parallel diffusive shock with velocity of $\sim 0.8 c$ (therefore favoring a mildly relativistic shock). The orbital dependency of the maximum electron energy for $\eta=10$ is shown in Fig. 3. During periods of low X-ray flux $E_{\mathrm{e}, \max }$ has a behaviour proportional to $t_{\mathrm{ad}}$ (see Fig. 2). During the outburst peaks, on the other hand, high energy losses are dominated by synchrotron and the cutoff is constant (following Eq. (6) for constant magnetic field). 


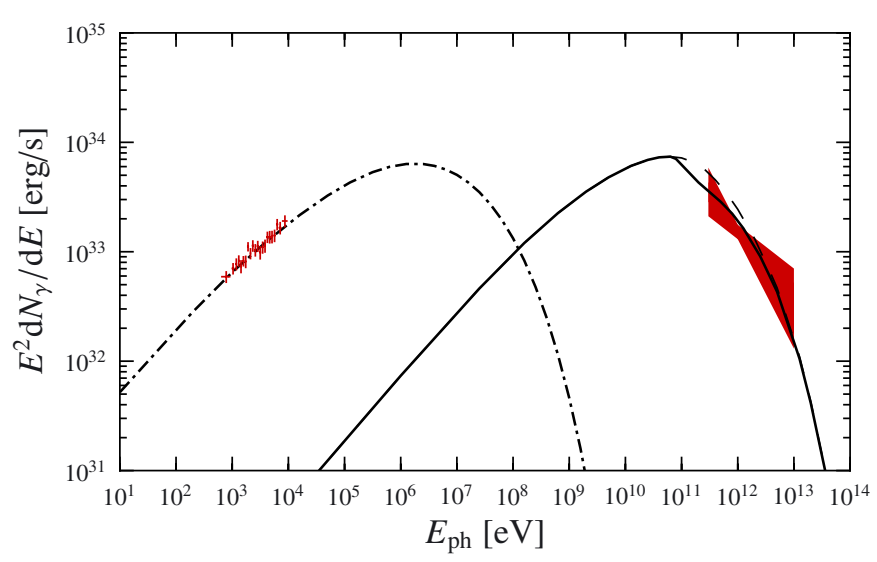

Fig. 4. Computed SED averaged over the three observation periods during the first outburst (phases $0.62,0.66$ and 0.70 ), with synchrotron (dot-dashed), IC (solid) and non-absorbed IC (dashed) components. The calculations were performed with $i=45^{\circ}$ and $B=0.22 \mathrm{G}$. The crosses show the XMM-Newton EPIC-pn spectrum averaged over three observations and deabsorbed taking $N_{\mathrm{H}}=5 \times 10^{21} \mathrm{~cm}^{-2}$. To compare the measured flux to the excess computed X-ray flux, we subtracted a pedestal power law spectrum corresponding to a $0.3-10 \mathrm{keV}$ flux of $11.5 \times 10^{-12} \mathrm{erg} \mathrm{cm}^{-2} \mathrm{~s}^{-1}$. The MAGIC simultaneous spectrum is shown as a red bow-tie.

\subsection{Synchrotron and IC emission}

For each position along the orbit, we calculate the emitted synchrotron spectrum from the evolved particle distribution and the magnetic field. Synchrotron emission in the X-ray range will be generally characterized by a photon index of $\Gamma_{\mathrm{X}}=\left(\alpha_{\mathrm{e}}+1\right) / 2 \approx$ 1.5. The orbital dependence of the electron maximum energy will affect the shape of the spectrum, typically hardening (softening) it for high (low) fluxes, which correspond to lower (higher) adiabatic losses and therefore a higher (lower) maximum electron energy. However, since the cutoff in the particle distribution is located at energies significantly higher than those responsible for X-ray emission, this effect is small in the X-ray energy band $\left(\Delta \Gamma_{\mathrm{X}} \sim 0.2\right)$.

We calculate the IC component of the spectrum using the anisotropic cross section and the stellar radiation field (blackbody radiation at $k T=2 \mathrm{eV}$ and $L_{\star}=10^{38} \mathrm{erg} \mathrm{s}^{-1}$ ) as the source of seed photons for the interaction. Following Eqs. (20) and (21) of Aharonian \& Atoyan (1981), the photon spectrum emitted by a population of isotropically distributed electrons can be described with a precision better than $3 \%$ (for $\epsilon \gg \epsilon_{0}$ and $\gamma \gg 1$ ) by

$$
\frac{\partial^{2} N(\theta, \epsilon)}{\partial \epsilon \partial \Omega} \simeq \frac{3}{16 \pi} c \sigma_{\mathrm{T}} \int_{\epsilon_{0, m}(\gamma, \theta)} \frac{n_{\epsilon_{0}}}{\epsilon_{0} \gamma^{2}} f\left(\epsilon, \epsilon_{0}, \theta, \gamma\right) \mathrm{d} \epsilon_{0},
$$

where $\epsilon$ and $\epsilon_{0}$ are the energies of the scattered and incident photons, respectively, in units of $m_{e} c^{2}, \theta$ is the scattering angle, $\sigma_{\mathrm{T}}$ is the Thomson cross section, $n_{\epsilon_{0}}$ is the number density of the stellar photon field,

$\epsilon_{0, m}(\gamma, \theta)=\frac{\epsilon}{2(1-\cos \theta) \gamma^{2}[1-\epsilon / \gamma]}$,

and

$f\left(\epsilon, \epsilon_{0}, \theta, \gamma\right)=1+\frac{z^{2}}{2(1-z)}-\frac{2 z}{b_{\theta}(1-z)}+\frac{2 z^{2}}{b_{\theta}^{2}(1-z)^{2}}$,

where $b_{\theta}=2(1-\cos \theta) \epsilon_{0} \gamma$ and $z=\epsilon / \gamma$. For a blackbody seed photon distribution, the shape of the resulting emitted spectrum

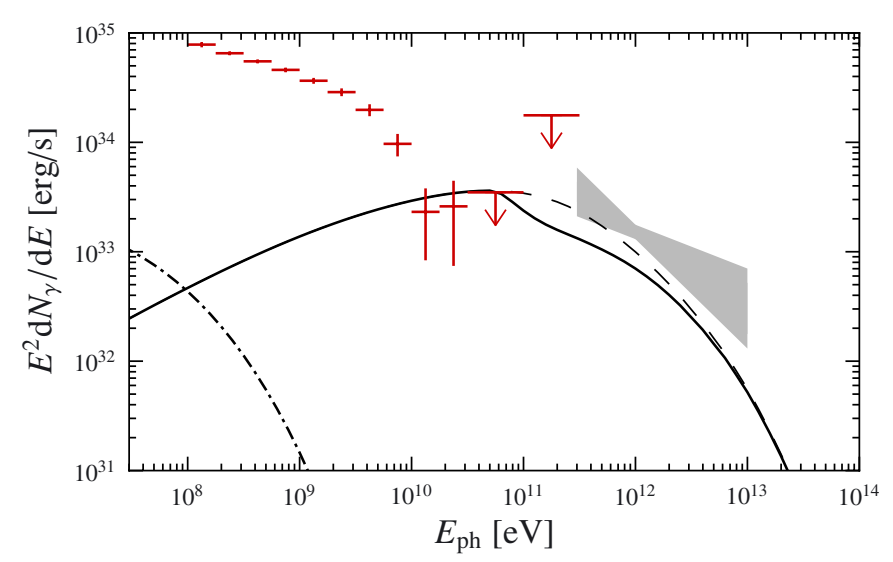

Fig. 5. Computed SED averaged over the whole orbit with synchrotron (dot-dashed), IC (solid) and non-absorbed IC (dashed) components, calculated with the same parameters as in Fig. 4. The crosses and upper limits indicate the phase-averaged Fermi/LAT spectrum. The MAGIC VHE spectrum during the outburst is shown as a bow-tie. It must be noted that the MAGIC spectrum corresponds to different phases than the computed SED and is shown as a reference to Fig. 4.

only depends on the parameter $b_{\theta}$ and the relativistic electron energy distribution.

\subsection{Pair production absorption}

The intense radiation field can also absorb very high energy $\gamma$-ray emission through pair production $\gamma \gamma \rightarrow \mathrm{e}^{+} \mathrm{e}^{-}$(Gould \& Schréder 1967). An exploration of the effects that this absorption can have on the observed spectrum of gamma-ray binaries can be found in Bosch-Ramon \& Khangulyan (2009) and references therein. The differential opacity for an emitted $\gamma$-ray of energy $\epsilon$ is given by

$\mathrm{d} \tau_{\gamma \gamma}=(1-\cos \psi) \sigma_{\gamma \gamma} n_{\epsilon_{0}} \mathrm{~d} l \mathrm{~d} \epsilon_{0}$,

where $l$ is the distance along the line of sight, $\epsilon_{0}$ is the energy of the stellar photon, $\psi$ is the interaction angle and the absorption cross section can be represented in the form:

$\sigma_{\gamma \gamma}=\frac{\pi r_{\mathrm{e}}^{2}}{2}\left(1-\beta^{2}\right)\left[2 \beta\left(\beta^{2}-2\right)+\left(3-\beta^{4}\right) \ln \left(\frac{1+\beta}{1-\beta}\right)\right]$,

where $\beta=(1-1 / s)^{1 / 2}$, and $s=\epsilon \epsilon_{0}(1-\cos \psi) / 2$. Pair production can only occur for $s>1$, when the centre of mass energy of the incoming photons is sufficiently high to create an electron positron pair. The cross section maximum takes place for $s \simeq$ 3.5 , with a value of $\sigma_{\gamma \gamma} \simeq 0.2 \sigma_{\mathrm{T}}$, and then decreases for $s \gg 1$.

The optical depth owing to pair production $\tau_{\gamma \gamma}$ is calculated at each point along the orbit by integrating the differential opacity (Eq. (10)) along the line of sight and over the blackbody seed photon distribution. The attenuation factor $\kappa=\exp \left(-\tau_{\gamma \gamma}\right)$ is applied to the intrinsic IC spectrum (dashed line in Fig. 4) to obtain the spectrum emitted out of the binary system (solid line in Fig. 4).

\section{Results}

As seen in Fig. 6, we were able to reproduce the X-ray and VHE light curves obtained during the 2007 multiwavelength campaign. Although the good agreement with the X-ray light curve is expected because we derived the dominant adiabatic losses 


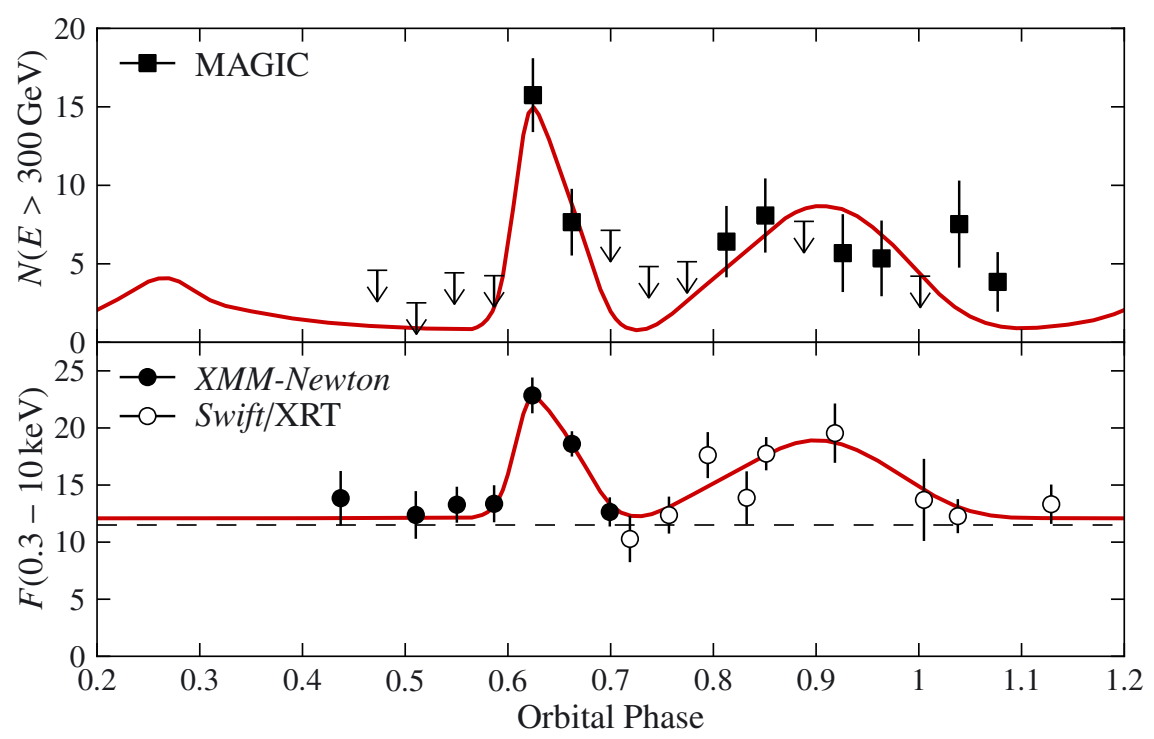

Fig. 6. Top: computed VHE light curve (red line) and observed VHE light curve by MAGIC in units of $10^{-12} \mathrm{ph} / \mathrm{cm}^{2} / \mathrm{s}$. Observations with a significance above $2 \sigma$ are shown in filled squares, while 95\% CL upper limits are shown otherwise. Bottom: computed X-ray light curve (red line) and X-ray light curve observed during the multiwavelength campaign with XMM-Newton (filled circles) and Swift/XRT (open circles) in units of $10^{-12} \mathrm{erg} / \mathrm{cm}^{2} / \mathrm{s}$. The pedestal flux is indicated by a horizontal dashed line. All error bars correspond to $1 \sigma$ uncertainties. The same parameters as in Fig. 4 are used for both panels. from the observed X-ray fluxes, the VHE light curve is a prediction of the model taking into account the binary geometry, the stellar photon field, and the non-thermal particle distribution. Furthermore, the spectral indices in the X-ray and VHE energy bands for the outburst at phases $0.6<\phi<0.7$ are well reproduced, as can be seen in Fig. 4.

\subsection{Electron population}

We found that the best fit to the X-ray and VHE photon indices can be obtained by taking a particle energy distribution with slope $\alpha_{\mathrm{e}}=2.1$. As mentioned above, the photon index in the $\mathrm{X}$-ray band directly depends on the particle index of the parent electron population. In the X-ray band, the observations show a photon index between $1.58 \pm 0.02$ and $1.66 \pm 0.02$ (plus a $1.85 \pm 0.02$ outlier) for the XMM-Newton observations, which is anti-correlated with the X-ray flux. The time-dependent energy cutoff given by the variability of cooling time scales provides a small time dependent variation of the photon index through a modification of the shape of the spectrum. Regarding the VHE observations, the measured photon index is $2.7 \pm 0.3_{\text {stat }} \pm 0.2_{\text {sys }}$ for the three observations during the first outburst. Because absorption is low at the orbital phases of the maximum, the photon index is determined by $\alpha_{\mathrm{e}}$ and the IC interaction angle. The parent particle population with $\alpha_{\mathrm{e}}=2.1$ results in computed X-ray photon indices in the range 1.55-1.67 and reproduces the observed anti-correlation between $\Gamma_{\mathrm{X}}$ and $F_{\mathrm{X}}$. In the VHE band the adopted $\alpha_{\mathrm{e}}$ results in photon indices in the range 2.6-2.8 for high-flux phases, whereas the spectrum is much softer for low flux phases because of the lower energy cutoff. Figure 4 presents the SED averaged over the phase ranges of the three simultaneous observations during the first outburst (phases 0.62, 0.66 and 0.70 ), and shows that both the flux levels and the photon indices at X-ray and VHE are well reproduced.

We found that the phase-averaged SED calculated using a power law with $\alpha_{\mathrm{e}}=2.1$ and a high-energy cutoff produces a flux too high in energies between 10 and $100 \mathrm{GeV}$ when compared to the phase-averaged spectrum measured by Fermi/LAT (Abdo et al. 2009). The GeV spectrum and fluxes along the orbit appear incompatible with one leptonic population. A way to avoid this excess flux is to consider the electron injection spectrum described by a broken power law with a harder particle index below $E_{\mathrm{e}}=4 \times 10^{11} \mathrm{eV}$. We found that the softest particle index for which the computed $\mathrm{GeV}$ spectrum is compatible with the Fermi/LAT measurement is $\alpha_{\mathrm{e}} \lesssim 1.8$. It must be noted that this change in the particle index at low energies does not affect the spectrum of particles that emit in the X-ray and VHE bands through synchrotron and IC, respectively. The Fermi/LAT spectrum along with the computed phase averaged SED is shown in Fig. 5. The phase-averaged Fermi/LAT cannot be easily compared with the peak spectrum shown in Fig. 4 because the X-ray/VHE and GeV measurements are not simultaneous. Furthermore, the orbital variability of the GeV spectrum at higher energies (i.e., the shape of the cutoff) has not been clearly established by Abdo et al. (2009), and a phase-resolved spectrum cannot be derived from a phase averaged-spectrum.

\subsection{Magnetic field}

The X-ray/VHE flux ratio is a very sensitive indicator of the magnetic field in the emitter. We found the magnetic field that best describes the observed light curves by performing a exploration of the parameter space of $B$ and the normalization of the injected particle spectrum $Q_{0}$. For each $\left(B, Q_{0}\right)$ pair we calculated the X-ray and VHE light curves and estimated the goodness-of-fit to the observed flux points using a $\chi^{2}$ test. The observed light curves are best described using an ambient magnetic field of $B=0.22 \mathrm{G}$. It is not trivial to set a formal uncertainty range on this value because of the unknown number of constraints that should be considered (e.g., the shape used for the adiabatic cooling curve). However, we see that for variations of the order of $0.05 \mathrm{G}$ around the best-fit value of $B=0.22 \mathrm{G}$ the observed X-ray and VHE light curves are no longer well described by the computed ones.

\subsection{Injection energy budget}

The adopted luminosity in injected accelerated particles is around $2 \times 10^{35} \mathrm{erg} \mathrm{s}^{-1}$ along the whole orbit. The computed emission bolometric luminosity, on the other hand, varies between $\sim 10^{34}$ and $\sim 1.5 \times 10^{35} \mathrm{erg} \mathrm{s}^{-1}$. Note that the adopted injected luminosity is only a lower limit because we arbitrarily chose the lowest ratio between the X-ray flux and the adiabatic cooling time scale. For higher adiabatic losses (while still proportional to X-ray emission), the emitted spectrum would be equivalent but the required injected luminosity would be higher.

The dominant energy band in the broad-band SED of LS I +61303 is the MeV-GeV region. Using the gamma-ray 
spectrum measured by Fermi/LAT (Abdo et al. 2009) we can obtain a lower limit on the power available for particle acceleration. The observed energy flux above $100 \mathrm{MeV}$ is $G_{100} \simeq$ $4.1 \times 10^{-10} \mathrm{erg} \mathrm{cm}^{-2} \mathrm{~s}^{-1}$, resulting in an observed luminosity of $2 \times 10^{35} \mathrm{erg} \mathrm{s}^{-1}$. Given this high luminosity, it is natural to think than the acceleration mechanism at play is able to provide a similar power to the X-ray and VHE emitting electrons.

\section{Discussion}

\subsection{Adiabatic time scales}

The orbital variability and range of adiabatic losses may be used to pose constraints on the hydrodynamical properties of the various scenarios proposed for LS I +61303. Hydrodynamical calculations have been performed in the binary pulsar scenario (Bogovalov et al. 2008; Khangulyan et al. 2008a) and for the interaction of a microquasar jet with the wind of a young star (Perucho \& Bosch-Ramon 2008; Perucho et al. 2010). However, for LS I +61303 it is difficult to calculate the adiabatic losses in each of the mentioned scenarios because of the uncertainties in the properties of the Be stellar wind and the relativistic outflows.

In our phenomenological framework, we find that adiabatic loss time scales range between 35 and 500 s, as seen in Fig. 2. Whereas it is difficult to relate these time scales to those arising from complex geometries (e.g. shocked pulsar wind or a microquasar jet), we can use the simple model of an expanding sphere to obtain insight on relevant emitter properties. The adiabatic loss time scale $t_{\mathrm{ad}}$ is related to the typical scale of the emitter $R_{\mathrm{em}}$ and its expansion velocity $v_{\text {exp }}$ through $t_{\text {ad }}=R_{\text {em }} / v_{\text {exp }}$. While the size of the emitting region is unknown, the fact that a one-zone emitter located at the compact object position can successfully explain the X-ray/VHE emission indicates that the region must be small compared with the orbital separation. Considering an emitting region with a radius $10 \%$ of the orbital separation we obtain a range of radii between $\sim 3 \times 10^{11} \mathrm{~cm}$ (at periastron) and $\sim 9 \times 10^{11} \mathrm{~cm}$ (at apastron). For these sizes, the maximum expansion velocities would be required for the shortest adiabatic cooling time scale, i.e. $35 \mathrm{~s}$. At apastron the expansion would need to be highly relativistic, with a velocity of $0.85 c$ (similar to the relativistic sound speed $c / \sqrt{3}$ ), corresponding to a Lorentz factor of $\Gamma=1.89$, whereas for periastron it would be mildly relativistic at $0.29 c$. For the longer adiabatic loss time scales of hundreds of seconds, the required expansion velocities would be much lower, of the order of $0.01 c-0.06 c$. A change in the size of the emitter could be caused by the change in external pressure from the stellar wind, resulting in a larger emitter at apastron (Takahashi et al. 2009). This would explain the location of the $\mathrm{X}$-ray/VHE outbursts around apastron, but it is not clear which magnetohydrodynamical process is responsible for the variation of the adiabatic loss time scale of more than an order of magnitude in $20 \mathrm{~h}$. Wind clumping can be considered as a source of pressure variability, as suggested by Zdziarski et al. (2010), but it would not explain the constant location of the X-ray/VHE outburst $(0.6<\phi<0.7)$ orbit after orbit. On the other hand, a transition of the emitter from a region dominated by polar wind to a region dominated by equatorial wind, or vice versa, could give rise to such a fast change in wind pressure. In addition, there could be other yet unknown properties of the stellar wind, as shown by the recent detection of a variable red shoulder in the $\mathrm{H} \alpha$ line by McSwain et al. (2010), which could be related to a tidal stream in the equatorial wind falling back onto the disk.

\subsection{Energetics}

The energetics of the system, regardless of the acceleration mechanism, are able to provide the required injected power used in our model given the high flux in the MeV-GeV band (see Sect. 4.3).

The pulsar-like spectrum of LS I +61303 in the $\mathrm{GeV}$ band (power law with exponential cutoff at a few $\mathrm{GeV}$ ) prompted Abdo et al. (2009) to consider a magnetospheric origin for the HE gamma-ray emission. In this scenario we can gain some insight on the spin-down luminosity of the putative pulsar from the HE gamma-ray luminosity. The observed energy flux $G_{100}$ from magnetospheric emission can be related to the emitted gammaray luminosity $L_{\gamma}$ as

$L_{\gamma}=4 \pi f_{\Omega}\left(\alpha, \zeta_{\mathrm{E}}\right) d^{2} G_{100}$,

where $f_{\Omega}$ is a beaming correction factor that depends on the geometry of the emitter, the magnetic angle $\alpha$ and the Earth viewing angle $\zeta_{\mathrm{E}}$. The emitted gamma-ray luminosity is related to the pulsar spin-down luminosity through the gamma-ray efficiency $\eta_{\gamma}=L_{\gamma} / \dot{E}$. A trend was found from EGRET observations that the efficiency scaled as $\dot{E}^{-1 / 2}$ (Thompson et al. 1999), and this was later confirmed (at least for $\dot{E}>10^{34} \mathrm{erg} \mathrm{s}^{-1}$ ) by Fermi/LAT arriving to $\eta_{\gamma} \simeq 0.034\left(\dot{E} / 10^{36} \mathrm{erg} \mathrm{s}^{-1}\right)^{-1 / 2}$ (Abdo et al. 2010). Using this relation, $\dot{E}$ can be inferred from $L_{\gamma}$ as $\dot{E} \simeq L_{\gamma}^{2} /\left(1.156 \times 10^{33} \mathrm{erg} \mathrm{s}^{-1}\right)$. From the LS I +61303 phaseaveraged gamma-ray spectrum measured by Abdo et al. (2009) we obtain $G_{100} \simeq 4.1 \times 10^{-10} \mathrm{erg} \mathrm{cm}^{-2} \mathrm{~s}^{-1}$, from which $L_{\gamma} \simeq$ $2 \times 10^{35} f_{\Omega} \mathrm{erg} \mathrm{s}^{-1}$. Following these considerations, the spin-down luminosity inferred from the Fermi/LAT observations is of $\dot{E} \simeq$ $3.3 \times 10^{37} f_{\Omega}^{2} \mathrm{erg} \mathrm{s}^{-1}$. Traditionally (e.g. polar cap models) it was assumed that the gamma-ray beam covers a solid angle of $1 \mathrm{sr}$ uniformly, so $f_{\Omega}=1 /(4 \pi) \simeq 0.08$, which would result in a spindown luminosity of $\dot{E} \simeq 2 \times 10^{35} \mathrm{erg} \mathrm{s}^{-1}$. However, more modern pulsar beaming models (e.g. slot gap and outer gap models) have $f_{\Omega} \sim 1$ for many viewing angles (Watters et al. 2009), which would result in a more energetic pulsar with $\dot{E} \simeq 3 \times 10^{37} \mathrm{erg} \mathrm{s}^{-1}$. The fact that the cutoff of the spectrum is located at high energies $\left(E_{\text {cutoff }}=6.3 \pm 1.1 \mathrm{GeV}\right)$ points to a high-altitude location for gamma-ray emission, since emission beyond a few $\mathrm{GeV}$ is very difficult close to the neutron star because of strong attenuation from $\gamma-B \rightarrow \mathrm{e}^{+} \mathrm{e}^{-}$absorption (Baring 2004). The general trend in the Fermi/LAT pulsar sample (Abdo et al. 2010) also points towards a high-altitude location, so we favour slot gap or outer gap models for which the spin-down luminosity of the putative pulsar in LS I +61303 would be of $\dot{E} \simeq 3 \times 10^{37} \mathrm{erg} \mathrm{s}^{-1}$. Even though the Fermi/LAT observations do not completely constrain the spin-down luminosity of the putative pulsar, the power available for particle acceleration is high. In the favoured scenario of $\dot{E} \simeq 3 \times 10^{37} \mathrm{erg} \mathrm{s}^{-1}$, assuming that the power in injected electrons constitutes only a fraction $10^{-2}$ of the spin-down luminosity (e.g. Sierpowska-Bartosik \& Torres 2008), the power in relativistic electron would be larger than our requirement of $L_{\text {inj }}=2 \times 10^{35} \mathrm{erg} \mathrm{s}^{-1}$.

Another possibility is that instead of a magnetospheric origin, the emission detected by Fermi/LAT has an Inverse Compton origin. In this case the efficiency between the spindown luminosity of the putative pulsar and the observed IC luminosity is expected to be of $1-10 \%$. Considering the observed luminosity of a few times $10^{35} \mathrm{erg} \mathrm{s}^{-1}$, the pulsar would need to have a spin-down luminosity of the order of $10^{37} \mathrm{erg} \mathrm{s}^{-1}$.

The higher values of spin-down luminosities pose a problem for the binary pulsar scenario regarding the shape of the detected radio emission. Dhawan et al. (2006) strongly argued that the 
rotating cometary-like tail they detected in VLBA observations was related to the shocked pulsar wind contained by the stellar wind. Romero et al. (2007) already questioned the ability of the stellar wind to contain the pulsar wind from the results of threedimensional hydrodynamical simulations even when considering a significantly lower spin-down luminosity of $10^{36} \mathrm{erg} \mathrm{s}^{-1}$. The shape of the interaction region between the stellar and pulsar winds is determined by the ratio of wind momentum fluxes

$\eta_{\mathrm{w}}=\frac{\dot{E}_{\mathrm{PSR}}}{\dot{M}_{\star} v_{\mathrm{w} \star} c}$,

where $\dot{M}_{\star}$ and $v_{\mathrm{w} \star}$ are the mass-loss rate and wind velocity of the Be star and $\dot{E}_{\mathrm{PSR}}$ is the spin-down luminosity of the pulsar. For the polar wind, we can take $\dot{M}_{\star}=10^{-8} M_{\odot} \mathrm{yr}^{-1}$ and $v_{\mathrm{w} \star}=10^{3} \mathrm{~km} \mathrm{~s}^{-1}$, resulting in a wind momentum flux ratio of $\eta_{\mathrm{w}}=0.53\left(\dot{E}_{\mathrm{PSR}} / 10^{36} \mathrm{erg} \mathrm{s}^{-1}\right)$ (Romero et al. 2007). Ignoring orbital motion (which on the other hand would give rise to small deviations in the inner region of the interaction, see Parkin \& Pittard 2008), the opening half-angle of the interaction cone would be

$\theta=180^{\circ} \frac{\eta_{\mathrm{w}}}{1+\eta_{\mathrm{w}}}$

as seen in analytical models of wind interaction (e.g. Antokhin et al. 2004). For a spin-down luminosity of $10^{36} \mathrm{erg} \mathrm{s}^{-1}$ the stellar wind would barely dominate the pulsar wind $\left(\eta_{\mathrm{w}}=0.53\right.$, $\theta=62^{\circ}$ ). Considering the favoured high-altitude location for gamma-ray production (i.e., slot gap or outer gap models) or an IC origin of the gamma-ray emission, the pulsar spin-down luminosity is of the order of $\dot{E}_{\mathrm{PSR}} \sim 10^{37} \mathrm{erg} \mathrm{s}^{-1}$. Given this luminosity, the pulsar wind would dominate the interaction and would not be contained by the stellar wind. The ratio of wind momentum fluxes would then be of $\eta_{\mathrm{w}} \simeq 16$ and the halfopening angle would approach $180^{\circ}$, posing a completely different scenario from that usually pictured by wind interaction models (e.g. Dubus 2006; Sierpowska-Bartosik \& Torres 2009; Zdziarski et al. 2010). A lower value of the beaming correction factor $f_{\Omega}$ in the magnetospheric scenario could mean that the pulsar spin-down luminosity is as low as $\dot{E}_{\mathrm{PSR}} \simeq 2 \times 10^{35} \mathrm{erg} \mathrm{s}^{-1}$ and thus contained by the stellar wind. However, given the high energy of the cutoff in the Fermi/LAT spectrum of LS I +61303 we favour a high-altitude location for gamma-ray production, leading to the higher value of the spin-down luminosity.

\subsection{Orbital inclination}

Synchrotron emission will have no dependence on the orbital inclination because we are assuming isotropic particle distribution and a fully disordered magnetic field. For IC emission, on the other hand, there is a dependence with the angle $\theta$ between the line of sight and the direction of propagation of the seed photons (i.e., the connecting line between the star and the compact object). Given the orbital configuration of LS I +61 303 (see Fig. 1), $\theta$ will not vary significantly with inclination for the phases around apastron, at which the source is detected in VHE. For $\gamma \gamma$ absorption, on the other hand, the dependency is on the angle between the directions of the VHE photon and the seed photon integrating along the line of sight out of the system. This means that absorption will show weaker orbital variability for low inclinations and stronger for high inclinations. For the flux peak observed at $\phi=0.62$, the optical depth of $\gamma \gamma$ absorption is practically independent of inclination at $\tau_{\gamma \gamma} \approx 0.2$ for $500 \mathrm{GeV}$ photons. However, the second, wider outburst at phases 0.8-1.1, does have an absorption dependency with inclination, with $\tau_{\gamma \gamma} \approx 1.4$ for $i=60^{\circ}$ but $\tau_{\gamma \gamma} \approx 0.3$ for $i=15^{\circ}$. The diminished absorption at low inclinations leads to a VHE flux higher than the observed values, particularly taking into account the upper limit measurement at phase 0.8. However, given the high uncertainties of the VHE nightly fluxes, the difference between the light curves computed for $i=60^{\circ}$ and $i=15^{\circ}$ is of less than $1 \sigma$. Even though we do not consider it significant given the present sensibilities, deeper VHE observations with firm detections at these phases could help to constrain the inclination of the orbit.

\subsection{Magnetic field}

The X-ray/VHE fluxes can be explained using a constant magnetic field of $B=0.22 \mathrm{G}$. A constraint on the accelerator maximum energy can be put by requiring that the Larmor radius of the accelerated electron $\left(r_{\mathrm{L}}=E_{\mathrm{e}} / q B c\right)$ is contained within the accelerator size. This translates to a maximum energy of $E_{\max }=300 B_{\mathrm{G}} R_{12} \mathrm{TeV}$, where $B_{\mathrm{G}}$ is the magnetic field in Gauss and $R_{12}$ the accelerator radius in units of $10^{12} \mathrm{~cm}$. For the magnetic field we found and considering an accelerator size of the order of $10 \%$ of the orbital separation, the maximum energy is well above the required $10 \mathrm{TeV}$ and radiative losses dominate at high energies (see Sect. 3.3). In the binary pulsar modelling approach of Dubus (2006), the magnetic flux was considered variable along the orbit as a consequence of the variation in the distance of the wind-shock region to the pulsar. In the shocked wind region, the magnetic field values obtained were as high as $10 \mathrm{G}$. Chernyakova et al. (2006) obtain a very similar magnetic field to ours, with $B=0.25 \mathrm{G}(B=0.35 \mathrm{G})$ for the low (high) flux state of the source. However, the model is based on an emitting particle spectrum very soft at high energies, resulting in a synchrotron component peaking in the radio band and an IC component dominating from soft $\mathrm{X}$-ray to the $\mathrm{MeV}$ band. They require another hadronic component to explain the VHE emission. The magnetic field in this model traces the flux ratio between radio and X-ray fluxes instead of ours, which traces the flux ratio between X-ray and VHE. On the other hand, in the microquasar approach used by Bosch-Ramon et al. (2006a), the magnetic field at the base of the jet was taken as $1 \mathrm{G}$. Most of these approaches use higher magnetic fields than the one we found to best reproduce the observational features. An explanation for this is likely the need in these models to generate both the pedestal and X-ray flux that we considered independently.

\section{Summary and concluding remarks}

We presented a radiation model of a single leptonic population that can successfully describe the data obtained through a simultaneous X-ray/VHE campaign on LS I +61303 performed by MAGIC in 2007. The observed X-ray/VHE correlation indicates both that the emission at these bands may come from a single particle population and that energy losses may be dominated by adiabatic losses. By assuming a constant magnetic field and injection along the orbit, we inferred the adiabatic loss rate from the X-ray light curve and the time dependent electron maximum energy as the balance between acceleration and energy loss time scales. We found that a quite efficient accelerator $(\eta \sim 10)$ is required to obtain the observed VHE spectra because of the fast adiabatic cooling. The injected electron spectrum is constant along the orbit and initially taken as a power law with a highenergy cutoff, but the Fermi/LAT HE spectrum poses a constraint on the hardness of the spectrum at lower energies, which we estimate has to be harder than $\alpha_{\mathrm{e}} \simeq 1.8$ for electron energies below 
$4 \times 10^{11} \mathrm{eV}$. At higher energies, on the other hand, an index of $\alpha_{\mathrm{e}}=2.1$ matches the observed X-ray and VHE photon indices. The observed light curves are best reproduced using a magnetic field of $B=0.22 \mathrm{G}$. The general picture shows that the observed emission in X-ray and VHE is fully compatible with originating in the same parent particle population under dominant adiabatic losses.

The required luminosity budget in injected electrons for the computed X-ray and VHE emission is $2 \times 10^{35} \mathrm{erg} / \mathrm{s}$. Both the microquasar and binary pulsar scenarios are able to provide these levels of luminosity in accelerated electrons. The GeV luminosity of LS I +61303 can also give us hints on the available power: independently of whether the detected emission is magnetospheric or IC emission, the injected power should be $\gtrsim 10^{37} \mathrm{erg} / \mathrm{s}$. If it is magnetospheric emission, the high-altitude favoured scenario of gamma-ray production implies that the spin-down luminosity of the pulsar is $\dot{E}=3 \times 10^{37} \mathrm{erg} / \mathrm{s}$. This high spindown luminosity poses problems for the binary pulsar scenario because the pulsar wind would not be contained by the stellar wind, which would give rise to a completely different scenario from the one usually considered.

The VHE and X-ray light curves of LS5039 and LS I +61 303 seem relatively similar, both peaking close to the apastron. For LS 5039 the maximum is also close to the inferior conjunction of the compact object, whereas this is not the case in LS I +61303. If the light curve maxima are related to apastron rather than to the observer orientation with respect to the system, it may point to a similar physical origin for the X-ray and VHE modulation in both sources. On the other hand, the adiabatic losses could have a different cause. Recall the very different stellar winds in both sources.

We found that the X-ray and the VHE emission are consistent with a single parent particle population, whereas the HE gamma-ray emission detected by Fermi/LAT probably has a different origin. The orbital anti-correlation and the exponential cutoff spectrum suggest that several non-thermal emitting regions may be present in the system. This could also explain the presence of a pedestal emission in X-ray that is compatible with constant emission along the orbit.

We conclude noting that simultaneous observations at the $\mathrm{X}$-ray and VHE bands provide a unique diagnostic tool to probe the emitter properties in galactic variable sources. Future observations, in particular by more sensitive next-generation VHE observatories, will allow us to better understand these peculiar systems.

Acknowledgements. We would like to thank Felix Aharonian and Dmitry Khangulyan for useful comments. We also thank the anonymous referee for constructive comments and suggestions that helped improve the manuscript. The authors acknowledge support of the Spanish MICINN under grant AYA200768034-C03-1 and FEDER funds. V.Z. was supported by the Spanish MEC through FPU grant AP2006-00077. V.B.-R. thanks the Max Planck Institut für Kernphysik for its kind hospitality and support. V.B.-R. also acknowledges the support of the European Community under a Marie Curie Intra-European fellowship.

\section{References}

Abdo, A. A., Ackermann, M., Ajello, M., et al. 2009, ApJ, 701, L123 Abdo, A. A., Ackermann, M., Ajello, M., et al. 2010, ApJS, 187, 460
Acciari, V. A., Beilicke, M., Blaylock, G., et al. 2008, ApJ, 679, 1427

Acciari, V. A., Aliu, E., Arlen, T., et al. 2009, ApJ, 700, 1034

Aharonian, F. A., \& Atoyan, A. M. 1981, Ap\&SS, 79, 321

Albert, J., Aliu, E., Anderhub, H., et al. 2006, Science, 312, 1771

Albert, J., Aliu, E., Anderhub, H., et al. 2009, ApJ, 693, 303

Anderhub, H., Antonelli, L. A., Antoranz, P., et al. 2009, ApJ, 706, L27

Antokhin, I. I., Owocki, S. P., \& Brown, J. C. 2004, ApJ, 611, 434

Aragona, C., McSwain, M. V., Grundstrom, E. D., et al. 2009, ApJ, 698, 514

Arnaud, K. A. 1996, in Astronomical Data Analysis Software and Systems V, ed. G. H. Jacoby, \& J. Barnes, ASP Conf. Ser., 101, 17

Baring, M. G. 2004, Adv. Space Res., 33, 552

Bogovalov, S. V., Khangulyan, D. V., Koldoba, A. V., Ustyugova, G. V., \& Aharonian, F. A. 2008, MNRAS, 387, 63

Bosch-Ramon, V., \& Khangulyan, D. 2009, Int. J. Mod. Phys. D, 18, 347

Bosch-Ramon, V., \& Paredes, J. M. 2004, A\&A, 425, 1069

Bosch-Ramon, V., Paredes, J. M., Romero, G. E., \& Ribó, M. 2006a, A\&A, 459, L25

Bosch-Ramon, V., Romero, G. E., \& Paredes, J. M. 2006b, A\&A, 447, 263

Casares, J., Ribas, I., Paredes, J. M., Martí, J., \& Allende Prieto, C. 2005, MNRAS, 360, 1105

Chernyakova, M., Neronov, A., \& Walter, R. 2006, MNRAS, 372, 1585

Dhawan, V., Mioduszewski, A., \& Rupen, M. 2006, in VI Microquasar Workshop: Microquasars and Beyond

Drury, L. O. 1983, Rep. Prog. Phys., 46, 973

Dubois, R. 2010, in Proceedings of the VERITAS Workshop on High Energy

Galactic Physics, Barnard College, Columbia University, New York, ed.

E. Aliu, J. Holder, \& R. Mukherjee, eConf C1005281, 9

Dubus, G. 2006, A\&A, 456, 801

Dubus, G., Cerutti, B., \& Henri, G. 2010, A\&A, 516, A18

Falcone, A. D., Grube, J., Hinton, J., et al. 2010, ApJ, 708, L52

Frail, D. A., \& Hjellming, R. M. 1991, AJ, 101, 2126

Ginzburg, V. L., \& Syrovatskii, S. I. 1964, The Origin of Cosmic Rays (MacMillan)

Gould, R. J., \& Schréder, G. P. 1967, Phys. Rev., 155, 1404

Gregory, P. C. 1999, ApJ, 520, 361

Gregory, P. C. 2002, ApJ, 575, 427

Grundstrom, E. D., Caballero-Nieves, S. M., Gies, D. R., et al. 2007, ApJ, 656, 437

Hinton, J. A., Skilton, J. L., Funk, S., et al. 2009, ApJ, 690, L101

Holder, J. 2010, in Proceedings of the VERITAS Workshop on High Energy Galactic Physics, Barnard College, Columbia University, New York, ed. E. Aliu, J. Holder, \& R. Mukherjee, eConf C1005281, 10

Jogler, T. 2009, Ph.D. Thesis, Technische Universität München

Kerschhaggl, M. 2011, A\&A, 525, A80

Khangulyan, D., Aharonian, F., Bogovalov, S., Koldoba, A., \& Ustyugova, G. 2008a, Int. J. Mod. Phys. D, 17, 1909

Khangulyan, D., Aharonian, F., \& Bosch-Ramon, V. 2008b, MNRAS, 383, 467

Khangulyan, D., Hnatic, S., Aharonian, F., \& Bogovalov, S. 2007, MNRAS, 380, 320

Maraschi, L., \& Treves, A. 1981, MNRAS, 194, 1P

Massi, M., \& Kaufman Bernadó, M. 2009, ApJ, 702, 1179

Massi, M., Ribó, M., Paredes, J. M., et al. 2004, A\&A, 414, L1

McSwain, M. V., Grundstrom, E. D., Gies, D. R., \& Ray, P. S. 2010, ApJ, 724, 379

Ong, R. A. 2010, The Astronomer's Telegram, 2948

Paredes, J. M. 1987, Ph.D. Thesis, University of Barcelona

Paredes, J. M., Marti, J., Peracaula, M., \& Ribo, M. 1997, A\&A, 320, L25

Paredes, J. M., Ribó, M., Bosch-Ramon, V., et al. 2007, ApJ, 664, L39

Parkin, E. R., \& Pittard, J. M. 2008, MNRAS, 388, 1047

Perucho, M., \& Bosch-Ramon, V. 2008, A\&A, 482, 917

Perucho, M., Bosch-Ramon, V., \& Khangulyan, D. 2010, A\&A, 512, L4

Rea, N., Torres, D. F., van der Klis, M., et al. 2010, MNRAS, 405, 2206

Romero, G. E., Okazaki, A. T., Orellana, M., \& Owocki, S. P. 2007, A\&A, 474, 15

Sidoli, L., Pellizzoni, A., Vercellone, S., et al. 2006, A\&A, 459, 901

Sierpowska-Bartosik, A., \& Torres, D. F. 2008, ApJ, 674, L89

Sierpowska-Bartosik, A., \& Torres, D. F. 2009, ApJ, 693, 1462

Takahashi, T., Kishishita, T., Uchiyama, Y., et al. 2009, ApJ, 697, 592

Thompson, D. J., Bailes, M., Bertsch, D. L., et al. 1999, ApJ, 516, 297

Watters, K. P., Romani, R. W., Weltevrede, P., \& Johnston, S. 2009, ApJ, 695, 1289

Zdziarski, A. A., Neronov, A., \& Chernyakova, M. 2010, MNRAS, 403, 1873 